\title{
Diagnose foliar e produção de soja após aplicação de resíduos em um latossolo sob sistema plantio direto
}

\section{Susiane Moura Cardoso dos Santos ${ }^{1}$, João Arthur Antonangelo², Angélica Cristina Fernandes Deus $^{3}$, Leonardo Theodoro Büll ${ }^{3}$}

\author{
${ }^{1}$ Universidade Estadual do Mato Grosso do Sul, Unidade Universitária de Cassilândia, Cassilândia, Mato Grosso do Sul, Brasil. E- \\ mail: susianemoura@yahoo.com.br \\ ${ }^{2}$ Oklahoma State University, Stillwater, Oklahoma, USA. E-mail: antonan@ ostatemail.okstate.edu \\ ${ }^{3}$ Universidade Estadual Paulista, Campus Botucatu, Botucatu, São Paulo, Brasil. E-mail: angeldeys@ hotmail.com , \\ bull@fca.unesp.br
}

Recebido: 11/06/2018; Aceito: 12/07/2018

\section{RESUMO}

A aplicação superficial de resíduos e o não revolvimento do solo decorrente do sistema plantio direto (SPD) favorecem os atributos químicos, físicos e biológicos do solo, afetando também a dinâmica de nutrientes ao longo do seu perfil. Além de atenuadores da acidez do solo, alguns resíduos ainda são excelentes fontes de nutrientes às plantas, dentre eles os macronutrientes, como o fósforo, o nitrogênio e o cálcio, e os micronutrientes, como o manganês e o zinco. Neste contexto, objetivou-se avaliar o estado nutricional e a produtividade de grãos da cultura da soja (Glycine max) cultivada sob SPD em um Latossolo Vermelho Distrófico submetido à aplicação superficial de resíduos industriais e urbanos. O delineamento experimental utilizado foi o de blocos casualizados, com quatro repetições $(\mathrm{n}=4)$. Os tratamentos foram constituídos por quatro resíduos: lodo de esgoto centrifugado e tratado com cal virgem (LC), lodo de esgoto de biodigestor (LB), escória de aciaria (E) e lama cal (Lcal), aplicados em quatro doses: 0 (controle), 2, 4 e $8 \mathrm{Mg} \mathrm{ha}^{-1}$. Houve aumento nos teores de N, P e Ca nas folhas da soja com a aplicação de doses crescentes dos resíduos. Já para o Mn e o Zn, os teores diminuíram com o aumento das doses de resíduos aplicadas, exceto para a dose $8 \mathrm{Mg} \mathrm{ha}^{-1}$. A aplicação crescente de doses de resíduos aumentou a produção de grãos na cultura da soja.

Palavras-chave: lodo de esgoto, lama cal, escória, Glycine max

\section{Leaf diagnosis and soybean yield after residues application in an oxisol under no-till}

\begin{abstract}
The surface application of residues and the non-soil revolving due to no-till (NT) favor the chemical, physical and biological soil attributes and affect the nutrients dynamics along its profile. In addition of being amendments of soil acidity, some residues are excellent sources of bioavailable nutrients, such as nitrogen, phosphorus and calcium as macronutrients, and zinc and manganese as micronutrients. In this context, we aimed to evaluate the nutritional status and grain yield of soybean (Glycine max) cultivated under NT in an Oxisol subjected to surface application of urban and industrial residues. The experimental design was the randomized block with four replications $(n=4)$. The treatments consisted of four residues: sewage sludge centrifuged and treated with virgin lime (LC), biodigestor sewage sludge (LB), steel slag (E) and lime sludge (Lcal), applied at four doses: 0 (control), 2, 4 and $8 \mathrm{Mg} \mathrm{ha}^{-1}$. There was an increase in the N, P and Ca contents in the soybean leaves. However, the $\mathrm{Mn}$ and $\mathrm{Zn}$ contents decreased as the doses of residues increased, except for the dose of $8 \mathrm{Mg} \mathrm{ha}^{-1}$. The increased doses of residues applied increased the grain yield in the soybean crop.
\end{abstract}

Key-words: sewage sludge, lime sludge, steel slag, Glycine max 


\section{Introdução}

O crescimento demográfico dos grandes centros urbanos caracteriza-se como a principal fonte geradora de diversos resíduos, domésticos e industriais. Estes, muitas vezes, são descartados no ambiente sem adequado tratamento. A utilização desses rejeitos, possibilitada por meio da reciclagem, faz com que sua adequada disposição torne-se um dos principais desafios a serem enfrentados pelos gestores ambientais (Nascimento et al., 2004a).

Dentre os resíduos utilizados, pode-se destacar o lodo de esgoto, um biossólido resultante do tratamento de águas residuárias, que apresenta potencial para utilização agrícola, já que sustenta quantidades significantes de matéria orgânica e de elementos essenciais para o desenvolvimento das plantas, podendo substituir, mesmo que parcialmente, os fertilizantes minerais. Dessa forma, o uso destes biossólidos pode desempenhar papel importante na produção agrícola e na manutenção da fertilidade do solo, sendo uma das alternativas mais promissoras para sua disposição (Nascimento et al., 2004b). Entretanto, a aplicação de altas doses de lodo esgoto pode aumentar a concentração de metais pesados no solo e nas folhas e grãos das plantas, prejudicando o meio ambiente (Silva et al., 2014).

Alguns resíduos industriais também podem ser utilizados pela agricultura como corretivos de acidez e fertilizantes, dentre estes destacam-se a lama cal e a escória de aciaria (Silva et al., 2014; Corrêa et al., 2007; 2008a). As melhorias nas características químicas do solo dada pela aplicação de escórias de siderurgia decorrem da elevação do $\mathrm{pH}$, da capacidade de troca catiônica (CTC) e da saturação por bases (V\%), bem como dos teores de $\mathrm{Ca}$ e $\mathrm{Mg}$, diminuindo ainda a acidez potencial $(\mathrm{H}+\mathrm{Al})$ (Prado; Fernandes, 2001; Prado et al., 2002). Os atributos químicos do solo supracitados ainda aumentam com a aplicação superficial de doses crescentes desses resíduos, com exceção à acidez potencial (Corrêa et al., 2007). Além do mais, o deslocamento de $\mathrm{Ca}$ e $\mathrm{Mg}$ à camadas mais profundas do solo também é favorecido, estando esses associados à uma maior quantidade de nitrato proporcionada pelo aumento das doses de aplicação dos próprios resíduos
(Corrêa et al., 2007). Portanto, seu potencial uso agrícola é como alternativa ao tradicional calcário, com a vantagem de que seu uso diminui o impacto ambiental em torno das indústrias produtoras de ferro e aço. Atualmente, é classificada como corretivo de acidez do solo, aplicando-lhe a mesma legislação do calcário para sua comercialização e utilização. (Prado et al., 2004).

Com isso, objetivou-se avaliar o estado nutricional e a produtividade de grãos da cultura da soja (Glycine max) cultivada sob sistema de plantio direto (SPD) em um Latossolo Vermelho Distrófico submetido à aplicação superficial de doses crescentes de resíduos industriais e urbanos

\section{Material e Métodos}

O trabalho foi conduzido a campo, no período de 2011 a 2013, na Fazenda Experimental Lageado, pertencente à Faculdade de Ciências Agronômicas FCA, Campus de Botucatu/UNESP, localizada no município de Botucatu-SP, na latitude de $22^{\circ} 51^{\prime} \mathrm{S}$, longitude de $48^{\circ} 26^{\prime} \mathrm{W}$ e altitude de $740 \mathrm{~m}$. O experimento foi conduzido em área manejada sob sistema plantio direto, submetido à aplicação superficial de resíduos industriais (escória de aciaria e lama cal) e urbanos (lodos de esgoto) no ano de 2002. Desde então, os resíduos foram aplicados seguindo as mesmas doses com intervalo de 1 ano, desta forma as reaplicações foram realizadas nos anos de 2005, 2007, 2009 e 2011. O solo da área experimental é classificado como Latossolo Vermelho Distrófico (Santos, 2013). O delineamento experimental utilizado foi o de blocos ao acaso, com quatro repetições $(n=4)$. Os tratamentos foram constituídos por quatro resíduos: LC - lodo de esgoto centrifugado com adição de cal virgem $(\mathrm{CaO})$, proveniente da Estação de Tratamento de Esgoto (ETE) de Presidente Prudente, São Paulo - SP; LB - lodo de esgoto proveniente de biodigestor com adição de polieletrólitos, produzido pela ETE de Barueri, SP; E escória de aciaria gerada pela Mannesmann, e Lcal lama cal proveniente da empresa de celulose Ripasa. As características químicas destes resíduos estão descritas na Tabela 1. As doses de 0 (controle), 2, 4 e $8 \mathrm{Mg} \mathrm{ha}^{-1}$ foram aplicadas de forma superficial e homogênea.

Tabela 1. Composição química dos resíduos: Lodo de esgoto centrifugado tratado com cal virgem (LC) e Lodo de esgoto de biodigestor (LB), Lama cal (Lcal) e Escória (E)

\begin{tabular}{|c|c|c|c|c|c|c|c|c|c|c|c|c|c|c|c|}
\hline Resíduo & $\mathrm{C} / \mathrm{N}$ & $\begin{array}{c}\mathrm{pH} \\
\mathrm{CaCl}_{2}\end{array}$ & Umidade & $\mathrm{MO}$ & $\mathrm{N}$ & $\begin{array}{l}\mathrm{P}_{2} \mathrm{O}_{5} \\
\ldots \ldots \%\end{array}$ & $\mathrm{~K}_{2} \mathrm{O}$ & $\mathrm{Ca}$ & $\mathrm{Mg}$ & $S$ & $\mathrm{Na}$ & $\mathrm{Cu}$ & $\begin{array}{c}\mathrm{Fe} \\
\mathrm{mg} \mathrm{kg}^{-1}\end{array}$ & $\mathrm{Mn}$ & $\mathrm{Zn}$ \\
\hline $\mathrm{LC}$ & $7 / 1$ & 8,1 & 25 & 10,5 & 0,8 & 0,4 & 0,1 & 12,1 & 0,2 & 0.1 & 270 & 75 & 4388 & 69 & 180 \\
\hline LB & $8 / 1$ & 6,8 & 18,9 & 42,1 & 3 & 3,4 & 0,1 & 1,9 & 0,4 & 0,7 & 243 & 99 & 17334 & 322 & 1176 \\
\hline Lcal & $93 / 1$ & 8,8 & 37,3 & 10,1 & 0,1 & 0,2 & 0,6 & 16,8 & 0,2 & 0 & 3263 & 6 & 265 & 91 & 9 \\
\hline E & $11 / 1$ & 12,4 & 10,0 & 2 & 0,1 & 1,2 & 0 & 21,8 & 2,4 & 0,9 & 684 & 23 & 145350 & 22455 & 25 \\
\hline
\end{tabular}

C/N: Relação Carbono/Nitrogênio

MO: Matéria Orgânica. 
O cultivar de soja 5D688 da Dow Agro Sciences foi semeado no dia 18 de novembro do ano agrícola 2011/2012 e no dia 22 de novembro do ano agrícola 2012/2013, seguindo as recomendações técnicas de acordo com os parâmetros pré-estabelecidos para cada cultura. A soja foi semeada sobre palha da aveia preta nos dois anos de cultivos. Utilizou-se densidade de semeadura de aproximadamente 22 sementes por metro, espaçamento de $0,45 \mathrm{~m}$ entre linha. Para o ano agrícola 2011/2012 e 2012/2013 a adubação foi realizada aplicando-se $300 \mathrm{~kg} \mathrm{ha}^{-1}$ dos fertilizantes formulados 420-20 e 0-20-20, respectivamente. As sementes foram tratadas com fungicida carboxina (carboxanilida) + tiram (dimetilditiocarbamato) (60 gi.a. por $100 \mathrm{~kg}$ de sementes) e inseticida tiametoxam (105 gi.a. por $100 \mathrm{~kg}$ de sementes) e inoculadas com Bradyrhizobium japonicum..

Para a diagnose foliar da soja, foram coletadas 10 folhas de forma aleatória em cada unidade experimental, na época de florescimento. Para tanto, foi destacada a terceira folha completamente desenvolvida, no sentido do ápice da haste para a base, seguindo metodologia descrita por Malavolta et al. (1997). As folhas foram secas em estufa de aeração forçada a $60{ }^{\circ} \mathrm{C}$ por $48 \mathrm{~h}$ e, em seguida, moídas para posterior análise química. Nas amostras das folhas diagnose foram determinados os teores de $\mathrm{N}, \mathrm{P}, \mathrm{K}, \mathrm{Ca}, \mathrm{Mg}, \mathrm{S}, \mathrm{B}, \mathrm{Cu}, \mathrm{Fe}, \mathrm{Mn}$, e Zn, conforme metodologia descrita por Malavolta et al., (1997).

A colheita da cultura da soja foi realizada em 29/03/2012, de forma mecanizada com colhedora de parcela, contendo três linhas centrais com sete metros de comprimento cada. Depois da trilha mecânica, os rendimentos de grãos de soja $\left(\mathrm{kg} \mathrm{ha}^{-1}\right)$ foram calculados com base na massa da amostra e na área coletada e ajustada para o teor de $130 \mathrm{~g} \mathrm{~kg}^{-1}$ de água (base úmida).

Os resultados foram submetidos à ANOVA através da análise de regressão, adaptando a melhor equação de acordo com teste $\mathrm{F}$ a $5 \%$, e ao teste de média entre os tratamentos, com a aplicação do teste Tukey a 5\%. Para tanto, utilizou-se programa Sisvar, versão 4.2.

\section{Resultados e Discussão}

Com exceção ao resíduo Escória (E), que apresentou um decréscimo no teor de $\mathrm{N}$ nas folhas com a maior dose aplicada (Tabela 2), verificou-se que os outros resíduos apresentaram comportamento crescente no ano de 2011/2012, opondo-se ao ano seguinte (2012/2013) (Tabela 3), onde os resultados do resíduo $\mathrm{E}$ se mantiveram com relação ao ano 2011/2012, enquanto que os demais decresceram com a maior dose do resíduo aplicada. Tal decréscimo decorrente do segundo ano de cultivo pode ser atribuído ao fato de a semeadura da soja ter ocorrido em curto período após aplicação superficial dos resíduos e do calcário. No entanto, o resíduo $\mathrm{E}$ foi o segundo a apresentar a maior média de $\mathrm{N}$ foliar. Assim como Miggiolaro et al. (2014), o menor teor de $\mathrm{N}\left(45,4\right.$ e $\left.36,0 \mathrm{~g} \mathrm{~kg}^{-1}\right)$ foi observado com a dose zero.

O resíduo Lodo de Biodigestor (LB) proporcionou menor valor médio $\left(46,9 \mathrm{~g} \mathrm{~kg}^{-1}\right)$ quando comparado ao calcário e aos resíduos Lodo Centrifugado (LC), Lama Cal (Lcal) e E nos dois anos. Malavolta (2006) infere que a multiplicação e a atividade dos microrganismos que decompõem os restos vegetais, transformando-os na matéria orgânica do solo exigem a assimilação de $\mathrm{N}$ mineral no protoplasma microbiano. Ou seja, quando restos culturais são incorporados ao solo, pode haver uma diminuição temporária no teor de $\mathrm{N}$ disponível da solução do solo, dessa forma podendo faltar $\mathrm{N}$ para a cultura seguinte. Neste estudo a soja foi semeada sobre palha da aveia preta nos dois anos de cultivos. A palha apresenta elevada relação $\mathrm{C} / \mathrm{N}$, condição que favorece a imobilização do N. De acordo com os resultados obtidos com a cultura da soja, após aplicação superficial dos resíduos e do calcário, pode-se constatar que os teores de $\mathrm{N}$ nas folhas, incluindo o pecíolo, estiveram dentro da faixa considerada adequada (40-54 $\mathrm{g} \mathrm{kg}^{-1}$ ), segundo Raij et al. (1997), até mesmo com a dose zero (0 kg ha$\left.{ }^{1}\right)$. A aplicação de calcário na dose recomendada de 2 $\mathrm{Mg} \mathrm{ha}{ }^{-1}$ proporcionou maior teor de $\mathrm{N}$ nas plantas no segundo ano de cultivo (Tabela 3).

$\mathrm{O}$ teor de $\mathrm{P}$ nas folhas foi pouco influenciado pela aplicação dos resíduos (Tabelas 2 e 3), assim como observado no estudo de Miggiolaro et al. (2014), onde os maiores valores de $\mathrm{P}$ também foram proporcionados pela aplicação do resíduo LC, mesmo não sendo o resíduo com maior teor de $\mathrm{P}$ em sua composição (Tabela 1). Diante disso, pode-se dizer que os lodos não apresentaram superioridade sobre os demais resíduos, que apresentam menor teor de $\mathrm{P}$ em sua composição. Além disso, a soja foi semeada utilizando-se $300 \mathrm{~kg} \mathrm{ha}^{-1}$ dos adubos formulados 4-20-20 (2011/2012) e 0-20-20 (2012/2013) à base de N-P-K nas duas safras, favorecendo os teores foliares de $\mathrm{P}$ nos dois anos de cultivo, e então não proporcionando grandes diferenças entre os resíduos. A faixa adequada para teor foliar de $\mathrm{P}$ na cultura da soja é de 2,5-5,0 $\mathrm{g} \mathrm{kg}^{-1}$ (Raij et al. 2001), dessa forma, o resíduo LC e a menor dose dos resíduos LC e E apresentaram valores inferiores ao adequado (Tabela 2). Provavelmente o $\mathrm{P}$ presente no solo não estava na forma mais disponível para as plantas mesmo considerando que todos os tratamentos proporcionaram teores de $\mathrm{P}$ superiores a dose zero. Solos de regiões tropicais possuem uma grande quantidade de óxidos de ferro $(\mathrm{Fe})$ e de alumínio ( $\mathrm{Al}$ ) na fração argila, minerais responsáveis pela adsorção e fixação de $\mathrm{P}$ previamente 
disponível na forma de ortofosfato- $\mathrm{H}_{2} \mathrm{PO}_{4}{ }^{-}$(Sample et al., 1980; Eriksson et al., 2015).

Nos dois anos agrícolas, a aplicação de calcário na dose recomendada de $2 \mathrm{Mg} \mathrm{ha}^{-1}$, proporcionou teor de $\mathrm{P}$ nas plantas acima do obtido no tratamento-controle (Tabelas 2 e 3). Vale lembrar que no primeiro ano de cultivo a média do calcário foi superior não apenas ao tratamento-controle como também à todos os demais tratamentos (Tabela 2).

Os maiores teores de K (Tabela 2 e 3 ) foram

Tabela 2. Valores de diagnose foliar de macronutrientes para o ano agrícola 2011/2012 após aplicação dos resíduos LB (lodo de biodigestor), LC (lodo centrifugado com adição de cal), Lcal (lama cal) e E (escória de aciaria) nas doses $0,2,4$ e 8 Mg ha- ${ }^{1}$

\begin{tabular}{|c|c|c|c|c|c|c|c|c|}
\hline \multicolumn{9}{|c|}{ Resíduos } \\
\hline \multicolumn{9}{|c|}{ Macro - Ano 2011/2012 } \\
\hline Dose & LB & $\mathrm{LC}$ & Lcal & $\mathrm{E}$ & LB & $\mathrm{LC}$ & Lcal & $\mathrm{E}$ \\
\hline $\mathrm{Mg} \mathrm{ha}^{-1}$ & \multicolumn{4}{|c|}{ 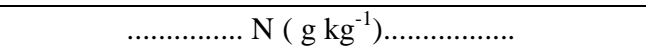 } & \multicolumn{4}{|c|}{$\ldots \ldots \ldots \ldots \ldots . \mathrm{P}\left(\mathrm{g} \mathrm{kg}^{-1}\right) \ldots \ldots \ldots \ldots \ldots \ldots$} \\
\hline 0 & 45,4 & 45,4 & 45,4 & 45,4 & 2,3 & 2,3 & 2,3 & 2,3 \\
\hline 2 & $46,5 \mathrm{c}$ & $51,1 \mathrm{ab}$ & $48,5 \mathrm{bc}$ & $52,3 \mathrm{a}$ & 2,5 & 2,4 & 2,6 & 2,4 \\
\hline 4 & $47,1 b$ & $51,2 \mathrm{a}$ & $49,6 \mathrm{ab}$ & $52,2 \mathrm{a}$ & $2,4 \mathrm{~b}$ & $2,5 \mathrm{ab}$ & $2,7 \mathrm{a}$ & $2,5 \mathrm{ab}$ \\
\hline 8 & $48,4 \mathrm{~b}$ & $55,0 \mathrm{a}$ & $50,7 \mathrm{~b}$ & $51,1 \mathrm{~b}$ & $2,4 \mathrm{~b}$ & $2,8 \mathrm{a}$ & $2,8 \mathrm{a}$ & $2,6 \mathrm{ab}$ \\
\hline Média & $46,9 b$ & $50,7 \mathrm{a}$ & $48,6 b$ & $50,3 a$ & $2,4 b$ & $2,5 \mathrm{ab}$ & $2,6 \mathrm{a}$ & $2,4 b$ \\
\hline Calcário & \multicolumn{4}{|c|}{50,0} & \multicolumn{4}{|c|}{3,0} \\
\hline & \multicolumn{4}{|c|}{$\ldots \ldots \ldots \ldots \ldots . . . \mathrm{K}\left(\mathrm{g} \mathrm{kg}^{-1}\right) \ldots \ldots \ldots \ldots \ldots \ldots$} & \multicolumn{4}{|c|}{$\ldots \ldots \ldots \ldots \ldots . . \mathrm{Ca}\left(\mathrm{g} \mathrm{kg}^{-1}\right) \ldots \ldots \ldots \ldots \ldots \ldots$} \\
\hline 0 & 15,2 & 15,2 & 15,2 & 15,2 & 5,6 & 5,6 & 5,6 & 5,6 \\
\hline 2 & 13,5 & 12,5 & 11,6 & 11,3 & $6,6 b$ & $7,7 \mathrm{~b}$ & $10,4 \mathrm{a}$ & $8,1 \mathrm{ab}$ \\
\hline 4 & 10,9 & 12,0 & 12,0 & 10,6 & $6,3 b$ & $10,2 \mathrm{a}$ & $10,3 \mathrm{a}$ & $10,2^{\mathrm{a}}$ \\
\hline 8 & 13,2 & 12,3 & 11,3 & 9,4 & $6,6 c$ & $9,3 \mathrm{~b}$ & $12,6 \mathrm{a}$ & $12,2^{\mathrm{a}}$ \\
\hline Média & 13,2 & 13,0 & 12,5 & 11,6 & $6,27 \mathrm{c}$ & $8,20 b$ & $9,73 a$ & $9,03 \mathrm{ab}$ \\
\hline Calcário & \multicolumn{4}{|c|}{10,0} & \multicolumn{4}{|c|}{9,0} \\
\hline
\end{tabular}

Tabela 3. Valores de diagnose foliar de macronutrientes para o ano agrícola 2012/2013 após aplicação dos resíduos LB (lodo de biodigestor), LC (lodo centrifugado com adição de cal), Lcal (lama cal) e E (escória de aciaria) nas doses 0, 2, 4 e $8 \mathrm{Mg} \mathrm{ha}^{-1}$

\begin{tabular}{|c|c|c|c|c|c|c|c|c|}
\hline \multicolumn{9}{|c|}{ Resíduos } \\
\hline \multicolumn{9}{|c|}{ Macro - Ano 2012/2013 } \\
\hline Dose & LB & LC & Lcal & $\mathrm{E}$ & LB & $\mathrm{LC}$ & Lcal & $\mathrm{E}$ \\
\hline $\mathrm{Mg} \mathrm{ha}^{-1}$ & \multicolumn{4}{|c|}{ 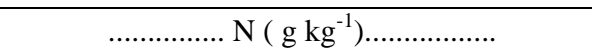 } & \multicolumn{4}{|c|}{ 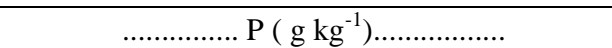 } \\
\hline 0 & 36,0 & 36,0 & 36,0 & 36,0 & 2,7 & 2,7 & 2,7 & 2,7 \\
\hline 2 & 44,4 & 43,7 & 45,9 & 46,9 & 2,8 & 3,0 & 3,0 & 2,9 \\
\hline 4 & 45,7 & 47,0 & 47,2 & 46,6 & 3,3 & 3,2 & 3,1 & 3,2 \\
\hline 8 & 40,5 & 44,5 & 43,4 & 46,3 & $2,8 b$ & $3,3 \mathrm{a}$ & $2,8 b$ & $3,0 \mathrm{~b}$ \\
\hline Média & 41,6 & 42,8 & 43,1 & 43,9 & 2,9 & 3,0 & 2,9 & 2,9 \\
\hline Calcário & \multicolumn{4}{|c|}{48,0} & \multicolumn{4}{|c|}{3,0} \\
\hline & \multicolumn{4}{|c|}{ 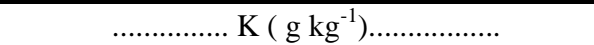 } & \multicolumn{4}{|c|}{ 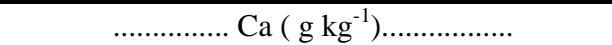 } \\
\hline 0 & 15,7 & 15,7 & 15,7 & 15,7 & 6,2 & 6,2 & 6,2 & 6,2 \\
\hline 2 & 14,4 & 13,0 & 13,2 & 13,4 & $7,4 \mathrm{c}$ & $13,0 \mathrm{a}$ & $9,9 b$ & $10,7 \mathrm{~b}$ \\
\hline 4 & 14,6 & 14,8 & 14,2 & 13,9 & $6,9 b$ & $11,0 \mathrm{a}$ & $11,6 \mathrm{a}$ & $12,1 \mathrm{a}$ \\
\hline 8 & 13,3 & 14,7 & 12,9 & 12,1 & $6,5 \mathrm{~b}$ & $11,5 \mathrm{a}$ & $13,1 \mathrm{a}$ & $12,8 \mathrm{a}$ \\
\hline Média & 14,5 & 14,6 & 14,0 & 13,8 & $6,8 \mathrm{~b}$ & $10,4 \mathrm{a}$ & $10,2 \mathrm{a}$ & $10,4 \mathrm{a}$ \\
\hline Calcário & \multicolumn{4}{|c|}{13,0} & \multicolumn{4}{|c|}{10,0} \\
\hline & \multicolumn{4}{|c|}{$\ldots . . \mathrm{Mg}\left(\mathrm{g} \mathrm{kg}^{-1}\right) \ldots \ldots \ldots \ldots \ldots \ldots \ldots \ldots \ldots$} & \multicolumn{4}{|c|}{$\ldots \ldots . . . \mathrm{S}\left(\mathrm{g} \mathrm{kg}^{-1}\right) \ldots \ldots$} \\
\hline 0 & 3,1 & 3,1 & 3,1 & 3,1 & 1,9 & 1,9 & 1,9 & 1,9 \\
\hline 2 & 3,7 & 4,0 & 3,1 & 3,5 & 2,2 & 2,0 & 2,3 & 2,1 \\
\hline 4 & $3,6 a$ & $3,5 \mathrm{a}$ & $2,3 b$ & $3,1 \mathrm{ab}$ & 2,0 & 2,4 & 2,2 & 2,4 \\
\hline 8 & $3,8 \mathrm{a}$ & $3,3 \mathrm{ab}$ & $2,6 \mathrm{~b}$ & $3,5 \mathrm{ab}$ & 1,9 & 2,1 & 2,1 & 2,2 \\
\hline $\begin{array}{c}\text { Média } \\
\text { Calcário }\end{array}$ & \multicolumn{4}{|c|}{4,0} & \multicolumn{4}{|c|}{2,0} \\
\hline
\end{tabular}


Para a cultura da soja, a faixa considerada adequada para teores foliares de K segundo Raij et al. (2001) é de 17$25 \mathrm{~g} \mathrm{~kg}^{1}$. Desse modo, pode-se dizer que todos os tratamentos estão abaixo da faixa considerada adequada para a cultura da soja. Para os teores de Ca, os resíduos Lcal e E apresentaram comportamento crescente em função das doses nos dois anos de cultivo, comportamento esse já esperado pelo fato de tais resíduos possuírem maior quantidade de $\mathrm{Ca}$ em sua composição (Tabela 1),. Os teores foliares adequados de Ca para a cultura da soja proposto por Raij et al. (2001) estão na faixa de $4-20 \mathrm{~g} \mathrm{~kg}$ 1 . Desse modo, nota-se que todos os tratamentos estão dentro da faixa adequada para os dois anos agrícolas, inclusive o controle (Tabelas 2 e 3). Os menores teores de Ca foram encontrados no tratamento controle, seguido do resíduo $\mathrm{LB}$, efeito esse justificado pela quantidade reduzida de Ca em sua composição (Tabelas 1 e 2).

Quanto aos teores de $\mathrm{Mg}$, provenientes do segundo ano de cultivo, observou-se que os maiores valores foram obtidos com a aplicação de LB (Tabela 3). Esse fato não era esperado uma vez que o resíduo E é o que possui maior quantidade de $\mathrm{Mg}$ em sua composição (Tabela 2).
Portanto, diante dos resultados, esse fato pode ser explicado pela relação $\mathrm{Ca} \times \mathrm{Mg}$, pois o resíduo LB possui baixos teores de $\mathrm{Ca}$, dessa forma houve maior absorção de $\mathrm{Mg}$, ocorrendo então o contrário com resíduos ricos em Ca. Essa menor disponibilidade do $\mathrm{Mg}$ no solo e, consequentemente, menor absorção pelas plantas faz com que a competição entre o Ca e o $\mathrm{Mg}$ pelos sítios de troca de adsorção dos colóides do solo favoreça o Ca em detrimento do Mg, fenômeno imposto pela lei das ações de massas. Há também a preferência pelo $\mathrm{Ca}$ em relação ao $\mathrm{Mg}$, de acordo com a série liotrópica, pois o primeiro apresenta menor raio iônico, o que confere maior afinidade à adsorção, mesmo ambos sendo cátions bivalentes (Fernandez et al., 2009).

Com relação aos micronutrientes, ainda para o primeiro ano de cultivo (Tabela 4), verificou-se as maiores médias foliares de Mn e Zn para o resíduo LB; com a aplicação dos outros resíduos houve diminuição dos valores de $\mathrm{Mn}$ foliar comparados com o tratamento controle (Tabela 4). Com exceção ao resíduo LB, as médias de $\mathrm{Zn}$ aumentaram no segundo ano (Tabela 5). Tal fato pode ser consequência da altíssima concentração de Zn do resíduo LB (Tabela 1).

Tabela 4. Valores de diagnose foliar de micronutrientes para o ano agrícola 2011/2012 após aplicação dos resíduos LB (lodo de biodigestor), LC (lodo centrifugado com adição de cal), Lcal (lama cal) e E (escória de aciaria) nas doses 0, 2, 4 e $8 \mathrm{Mg}^{-1}$

\begin{tabular}{|c|c|c|c|c|c|c|c|c|}
\hline \multicolumn{9}{|c|}{ Resíduos } \\
\hline \multicolumn{9}{|c|}{ Micronutrientes - Ano 2011/2012 } \\
\hline Dose & LB & $\mathrm{LC}$ & Lcal & $\mathrm{E}$ & LB & $\mathrm{LC}$ & Lcal & $\mathrm{E}$ \\
\hline $\mathrm{Mg} \mathrm{ha}^{-1}$ & \multicolumn{4}{|c|}{........ Mn $\left(\mathrm{mg} \mathrm{kg}^{-1}\right) \ldots \ldots \ldots \ldots \ldots \ldots$} & \multicolumn{4}{|c|}{$\mathrm{Zn}\left(\mathrm{mg} \mathrm{kg}^{-1}\right) \ldots \ldots \ldots \ldots \ldots \ldots$} \\
\hline 0 & 105,7 & 105,7 & 105,7 & 105,7 & 54,7 & 54,7 & 54,7 & 54,7 \\
\hline 2 & 152,7 & 73,0 & 80,7 & 66,7 & $82,3 a$ & $48,0 \mathrm{~b}$ & $42,7 b$ & $38,3 \mathrm{~b}$ \\
\hline 4 & 93,7 & 68,0 & 50,7 & 55,7 & $99,0 \mathrm{a}$ & $45,7 \mathrm{~b}$ & $37,3 b$ & $34,0 \mathrm{~b}$ \\
\hline 8 & $333,3 \mathrm{a}$ & $48,7 \mathrm{~b}$ & $50,7 \mathrm{~b}$ & $67,3 \mathrm{~b}$ & $183,7 \mathrm{a}$ & $34,3 b$ & $36,3 b$ & $28,7 \mathrm{~b}$ \\
\hline Média & $171,3 a$ & $73,8 \mathrm{~b}$ & $71,9 \mathrm{~b}$ & $73,8 b$ & $104,9 a$ & $45,7 b$ & $42,8 b$ & $38,9 \mathrm{~b}$ \\
\hline Calcário & \multicolumn{4}{|c|}{47,0} & \multicolumn{4}{|c|}{35,0} \\
\hline
\end{tabular}

Tabela 5. Valores de diagnose foliar de micronutrientes para o ano agrícola 2012/2013 após aplicação dos resíduos LB (lodo de biodigestor), LC (lodo centrifugado com adição de cal), Lcal (lama cal) e E (escória de aciaria), em função nas doses $0,2,4$ e $8 \mathrm{Mg}$ $\mathrm{ha}^{-1}$

\begin{tabular}{|c|c|c|c|c|c|c|c|c|}
\hline \multicolumn{9}{|c|}{ Resíduos } \\
\hline \multicolumn{9}{|c|}{ Micro - Ano 2012/2013 } \\
\hline Dose & LB & $\mathrm{LC}$ & Lcal & E & LB & $\mathrm{LC}$ & Lcal & $\mathrm{E}$ \\
\hline $\mathrm{Mg} \mathrm{ha}^{-1}$ & \multicolumn{4}{|c|}{ ……...... B $\left(\mathrm{mg} \mathrm{kg}^{-1}\right) \ldots \ldots \ldots \ldots \ldots \ldots$} & \multicolumn{4}{|c|}{$\ldots \ldots \ldots \ldots \ldots . . . \mathrm{Fe}\left(\mathrm{mg} \mathrm{kg}^{-1}\right) \ldots \ldots \ldots \ldots \ldots \ldots$} \\
\hline 0 & 36,0 & 36,0 & 36,0 & 36,0 & 146,3 & 146,3 & 146,3 & 146,3 \\
\hline 2 & 36,8 & 36,2 & 36,5 & 37,1 & 139,7 & 159,0 & 151,0 & 131,3 \\
\hline 4 & $37,5 \mathrm{ab}$ & $35,5 \mathrm{bc}$ & $32,1 \mathrm{c}$ & $40,6 \mathrm{a}$ & $135,7 b$ & $154,7 b$ & $177,0 \mathrm{~b}$ & $220,7 \mathrm{a}$ \\
\hline 8 & 39,1 & 35,2 & 35,8 & 39,6 & $138,3 \mathrm{~b}$ & $176,0 \mathrm{ab}$ & $182,7 \mathrm{a}$ & $145,3 \mathrm{ab}$ \\
\hline Média & $37,4 \mathrm{ab}$ & $35,7 b c$ & $35,1 \mathrm{c}$ & $38,3 \mathrm{a}$ & $140,0 \mathrm{~b}$ & $159,0 \mathrm{ab}$ & $164,2 \mathrm{a}$ & $160,9 \mathrm{ab}$ \\
\hline Calcário & \multicolumn{4}{|c|}{36,5} & \multicolumn{4}{|c|}{161,0} \\
\hline & \multicolumn{4}{|c|}{ 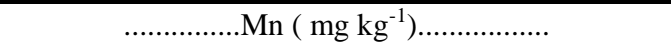 } & \multicolumn{4}{|c|}{$\ldots \ldots \ldots \ldots \ldots . . . . \mathrm{Zn}\left(\mathrm{mg} \mathrm{kg}^{-1}\right) \ldots \ldots \ldots \ldots \ldots \ldots \ldots$} \\
\hline 0 & 147,7 & 147,7 & 147,7 & 147,7 & 76,7 & 76,7 & 76,7 & 76,7 \\
\hline 2 & 100,0 & 73,3 & 55,3 & 65,7 & $80,7 \mathrm{a}$ & $72,3 \mathrm{ab}$ & $45,3 \mathrm{c}$ & $50,7 \mathrm{bc}$ \\
\hline 4 & 75,3 & 63,7 & 65,3 & 60,0 & $92,3 \mathrm{a}$ & $69,0 \mathrm{ab}$ & $38,7 \mathrm{c}$ & $49,3 b c$ \\
\hline 8 & $203,7 \mathrm{a}$ & $54,0 \mathrm{~b}$ & $66,3 b$ & $53,6 \mathrm{~b}$ & $147,3 \mathrm{a}$ & $45,3 \mathrm{~b}$ & $39,7 b$ & $37,0 \mathrm{~b}$ \\
\hline Média & $131,7 \mathrm{a}$ & $84,7 b$ & $83,7 b$ & $81,7 \mathrm{~b}$ & $99,3 \mathrm{a}$ & $65,8 b$ & $50,0 \mathrm{c}$ & $53,4 \mathrm{bc}$ \\
\hline Calcário & \multicolumn{4}{|c|}{57,3} & \multicolumn{4}{|c|}{41,7} \\
\hline
\end{tabular}


Além do $\mathrm{Mn}$ e $\mathrm{Zn}$ que tiveram incrementos significativos no primeiro ano (Tabela 4), os teores de boro (B) e ferro (Fe) foliares também apresentaram alterações em função das aplicações dos resíduos (Tabela 5). Os resíduos aplicados, principalmente o LB, possuem altos teores de $\mathrm{Fe}$ em sua composição, o que provavelmente favoreceu a sua absorção.

Quanto aos teores foliares de $\mathrm{N}$, com exceção do resíduo $\mathrm{E}$, que teve ajuste quadrático, os demais se ajustaram linearmente e de forma crescente (Figura 1). Os resíduos LC, Lcal e E apresentam efeito significativo e ajuste linear para os teores de P (Figura 1). O valor médio de $\mathrm{P}$ observado com a aplicação do calcário foi $3,0 \mathrm{~g} \mathrm{~kg}^{-1}$, superior a todos os valores observados. De forma geral, incrementos lineares nos teores de $\mathrm{N}$ e $\mathrm{P}$ em folhas de soja cultivada com lodo de esgoto de biodigestor foram destacados (Figura 1), sendo tais resultados também observados por Corrêa et al. (2008b) durante três anos consecutivos.

O K não apresentou efeito significativo, obtendo-se apenas ajuste quadrático com os valores das médias (Figura 1). Para o Ca os resíduos LC, Lcal e E, o ajuste à equação foi quadrático, porém para os resíduos Lcal e E não foi possível observar o ponto de decréscimo (Figura 1). Na Figura 2, o efeito do lodo de esgoto sobre os teores de $\mathrm{K}$ apresentou ajuste linear decrescente em função das doses dos resíduos. Concluise que o uso dos resíduos diminuiu os teores foliares de $\mathrm{K}$, não alcançando a média considerada adequada para a cultura da soja (17-25 $\mathrm{g} \mathrm{kg}^{-1}$ ) de acordo com Raij et al. 2001. Destaca-se novamente que os resíduos aplicados
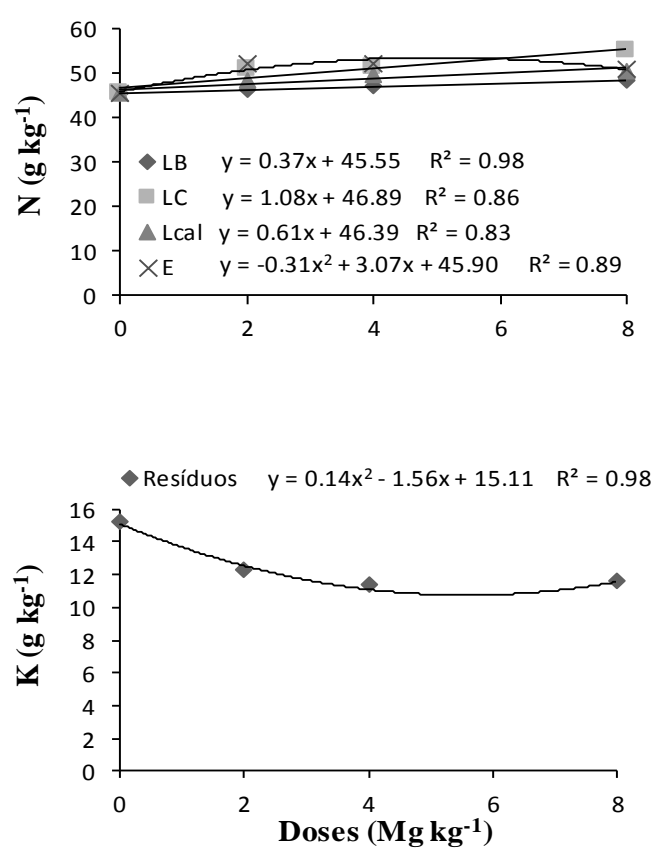

Figura 1. Regressão linear para os teores de macronutrientes de acordo com as doses de resíduos aplicadas $\left(0,2,4\right.$ e $\left.8 \mathrm{Mg}^{-1}\right)$ na safra agrícola 2011/2012 são ricos em $\mathrm{Ca}$, dessa forma a absorção de $\mathrm{K}$ pelas plantas foi prejudicada.

A aplicação das doses do resíduo LB proporcionou os maiores teores de $\mathrm{Zn}$ nas folhas nos dois anos agrícolas, 2011/2012 e 2012/2013 (Figuras 3 e 4). Opostamente verificou-se redução dos teores foliares de $\mathrm{Zn}$ quando os demais resíduos foram aplicados, principalmente o LC e o E, também em ambas as safras (Figuras 3 e 4). Nos dois anos de cultivo, a aplicação dos resíduos e do calcário proporcionou maior produtividade de grãos de soja quando comparado ao tratamento controle (dose $0,0 \mathrm{Mg} \mathrm{ha}^{-1}$ ), sendo o maior valor observado com a aplicação de $2 \mathrm{Mg} \mathrm{ha}^{-1}$ do resíduo LB (2834 $\left.\mathrm{kg} \mathrm{ha}^{-1}\right)$ para o primeiro ano e com a aplicação de $2 \mathrm{Mg} \mathrm{ha}^{-1}$ do resíduo E (3595 $\mathrm{kg} \mathrm{ha}^{-1}$ ) para o segundo ano (Tabela 6). Resultados semelhantes foram observados por Miggiolaro et al. (2014) na mesma área experimental, com a aplicação dos mesmos resíduos.

De forma geral, a produtividade de grãos de soja aumentou em função da aplicação das doses dos resíduos, sendo o mesmo observado por Corrêa et al (2009). Portanto, a aplicação superficial desses resíduos em SPD pode ser uma alternativa para aumentar a produtividade da cultura da soja. Nesse caso, deve-se destacar a escória de aciaria, lodo de esgoto centrifugado e lama cal, por ainda não impactarem negativamente o meio ambiente, uma vez que sejam atendidas as diretrizes de utilização na resolução do CONAMA 375 (2010) para lodo de esgoto e a lei NBR 10004 (ABNT 1987) para resíduos industriais (Andrade et. al 2010).
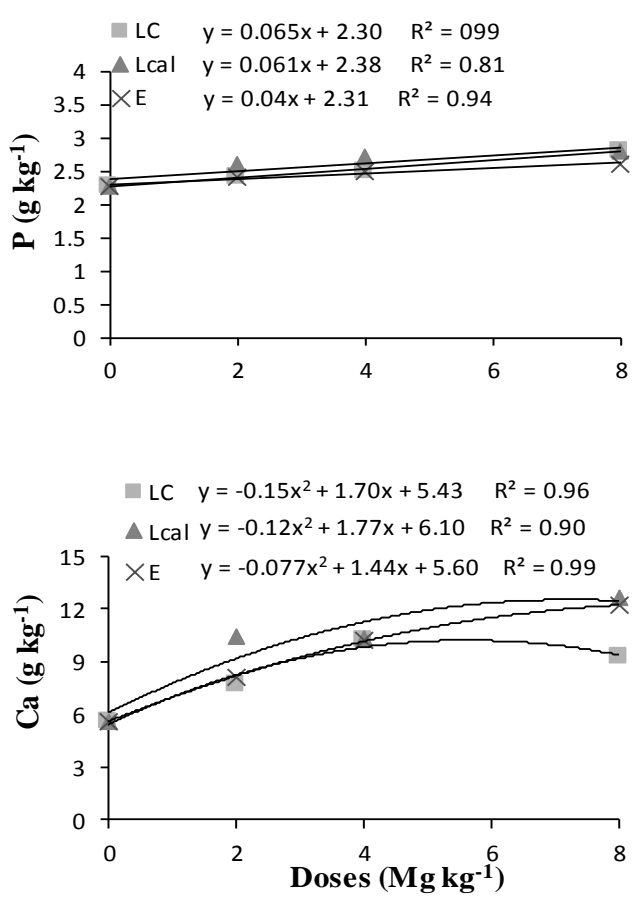

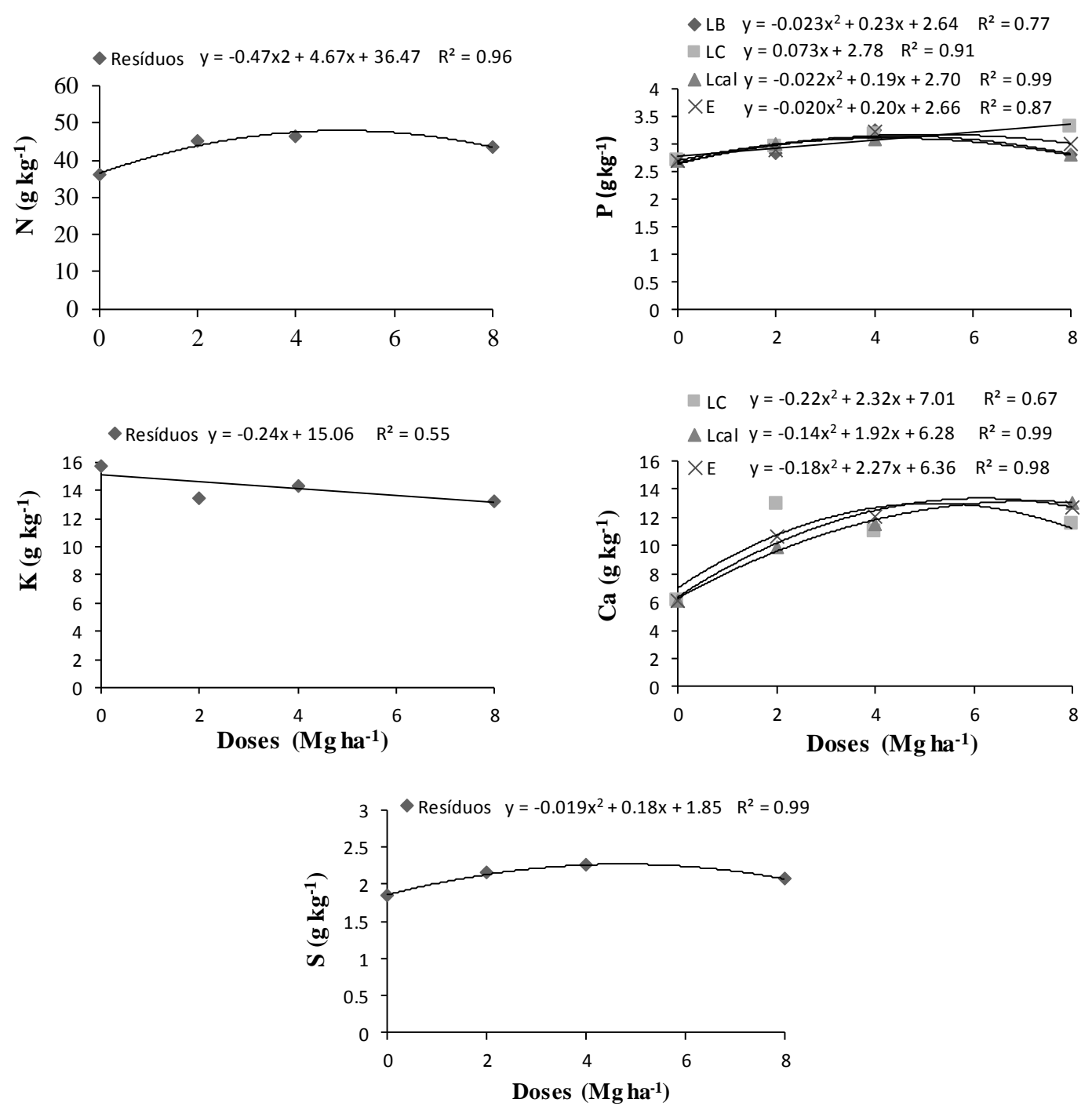

Figura 2. Regressão linear para os teores de macronutrientes de acordo com as doses de resíduos aplicadas $\left(0,2,4\right.$ e $\left.8 \mathrm{Mg}^{-1}\right)$ na safra agrícola 2012/2013.

As produções médias dos tratamentos $\mathrm{LC}$, Lcal e E foram superiores ao obtido com a aplicação do resíduo LB (2192 e $2418 \mathrm{~kg} \mathrm{ha}^{-1}$ para as safras 2011/2012 e 2012/2013, respectivamente), sendo então os menores valores observados com a aplicação desse resíduo na dose de $8 \mathrm{Mg} \mathrm{ha}^{-1}$ em comparação a aplicação dos outros resíduos nessa mesma dose (Tabela 6). Porém, mesmo com decréscimo na produtividade, não foram observados sintomas de deficiência.

Tais resultados justificam a aplicação superficial dos resíduos LB, LC, Lcal e E em SPD como alternativa aos fertilizantes comerciais e calcário para se obter boa produtividade de soja sem causar impacto ao meio ambiente. A produção média dos tratamentos LC, Lcal e E foi superior à obtida com a aplicação do resíduo LB (2191,9), sendo o menor valor observado com a aplicação do mesmo na dose de $8 \mathrm{Mg} \mathrm{ha}^{-1}(1520,8)$. Desta forma, evidencia-se que a aplicação do resíduo LB elevou a produção da soja até a dose de $4 \mathrm{Mg} \mathrm{ha}^{-1}$ aplicada, no entanto, com a maior dose $\left(8 \mathrm{Mg} \mathrm{ha}^{-1}\right)$ houve decréscimo significativo, provavelmente causado pelo alto teor de Zn presente neste resíduo.

Para o primeiro ano (2011/2012), os resíduos não apresentaram efeito significativo com relação à produtividade de grãos de soja, sendo apresentadas na Figura 5 apenas as médias em função das doses dos resíduos aplicadas. Ao ajustar-se à regressão quadrática é possível dizer que a dose de $4,58 \mathrm{Mg}^{-}{ }^{-}$ ${ }^{1}$ proporcionou maior produtividade; $2800 \mathrm{~kg} \mathrm{ha}^{-1}$, valor superior a média brasileira nos últimos 40 anos de 2,482 $\mathrm{kg} \mathrm{ha}^{-1}$ segundo a Conab (2016).

Em relação ao segundo ano de cultivo (2012/2013), todos os resíduos apresentaram efeito significativo e ajuste quadrático, com exceção da Lcal, que apresentou-se de forma linear não sendo possível realizar o ajuste para saber qual a melhor dose de aplicação (Figura 5). 

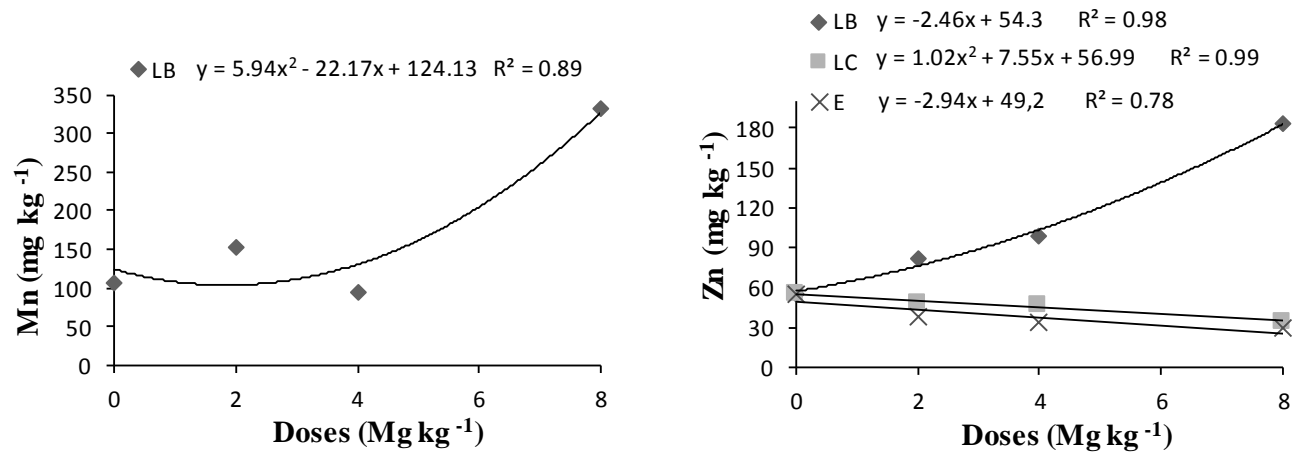

Figura 3. Regressão linear para os teores de micronutrientes de acordo com as doses de resíduos aplicadas $\left(0,2,4\right.$ e $\left.8 \mathrm{Mg}^{-1}\right)$ na safra agrícola 2011/2012
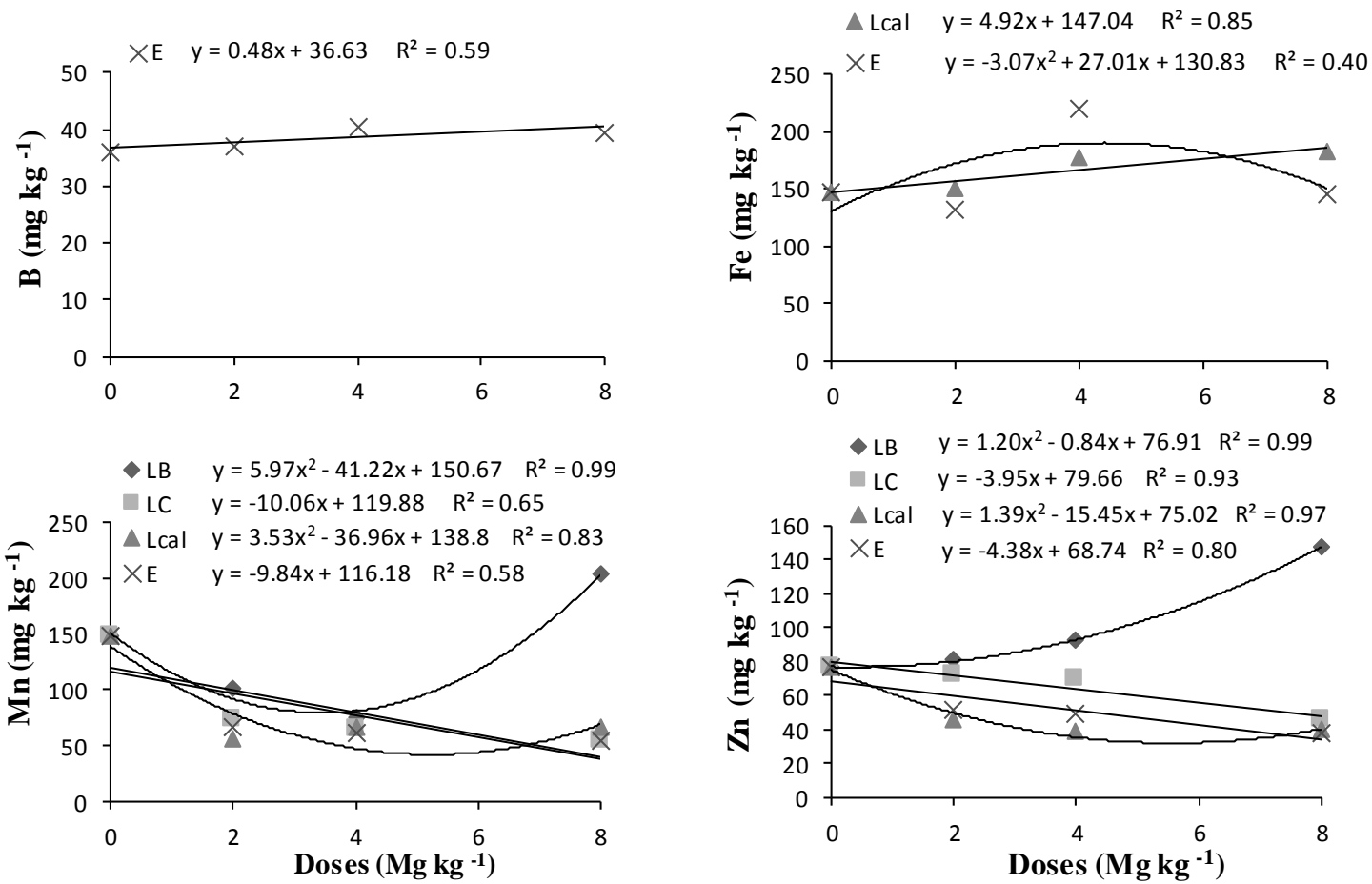

Figura 4. Regressão linear para os teores de micronutrientes de acordo com as doses de resíduos aplicadas $\left(0,2,4\right.$ e $\left.8 \mathrm{Mg}^{-1}\right)$ na safra agrícola 2012/2013.

Tabela 6. Produtividade $\left(\mathrm{kg} \mathrm{ha}^{-1}\right.$ ) de grãos da soja para as safras de 2011/2012 e 2012/2013 após aplicação dos resíduos LB (lodo de biodigestor), LC (lodo centrifugado com adição de cal), Lcal (lama cal) e E (escória de aciaria) nas doses 0, 2, 4 e $8 \mathrm{Mg} \mathrm{ha}^{-1}$

\begin{tabular}{|c|c|c|c|c|c|c|c|c|c|}
\hline Dose & \multicolumn{4}{|c|}{ Ano $2011 / 2012$} & Produção & \multicolumn{4}{|c|}{ Ano $2012 / 2013$} \\
\hline $\mathrm{Mg} \mathrm{ha}^{-1}$ & LB & LC & Lcal & $\mathrm{E}$ & $\mathrm{kg} \mathrm{ha}^{-1}$ & LB & $\mathrm{LC}$ & Lcal & $\mathrm{E}$ \\
\hline 0 & 1714,0 & 1714,0 & 1714,0 & 1714,0 & & 2352,8 & 2352,8 & 2352,8 & 2352,8 \\
\hline 2 & 2653,3 & 2834,0 & 2541,3 & 2394,2 & & 2574,5 & 3467,6 & 3403,2 & 3595,3 \\
\hline 4 & 2742,5 & 2698,7 & 2495,5 & 2793,8 & & 3210,8 & 3537,7 & 3013,4 & 3425,6 \\
\hline 8 & $2721,1^{\mathrm{a}}$ & $1520,8 b$ & $2517,5 \mathrm{a}$ & $2198,0 \mathrm{ab}$ & & $1535,5 \mathrm{~b}$ & $3301,0 \mathrm{a}$ & $3285,6 \mathrm{a}$ & $3189,8 \mathrm{a}$ \\
\hline Média & 2191,9 & 2457,7 & 2317,1 & 2275,0 & & $2418,4 b$ & $3164,8^{a}$ & $3013,7 \mathrm{a}$ & $3140,9 \mathrm{a}$ \\
\hline Calcário & \multicolumn{4}{|c|}{2331,7} & & \multicolumn{4}{|c|}{3203,67} \\
\hline
\end{tabular}

Médias seguidas de mesma letra minúscula (na linha) não diferem entre si pelo teste de Tukey a 5\%. 

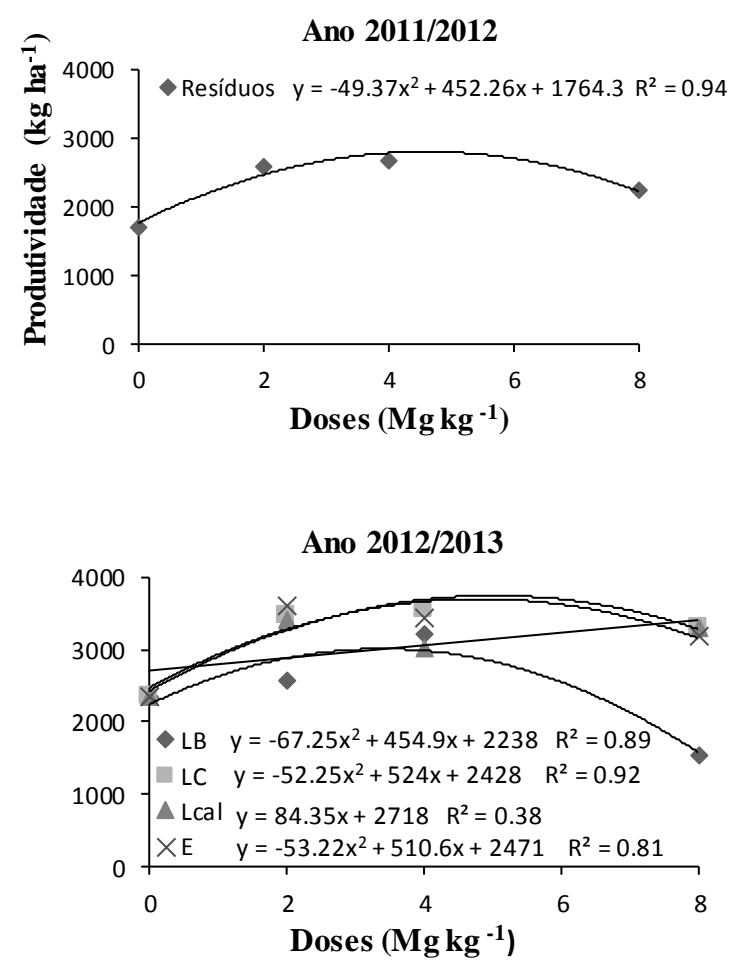

Figura 5. Regressão linear para a produtividade de grãos da soja de acordo com as doses de resíduos aplicadas $(0,2,4$ e 8 $\mathrm{Mg} \mathrm{ha}^{-1}$ ) nas safras agrícolas 2011/2012 e 2012/2013

\section{Conclusões}

Todos os resíduos utilizados proporcionaram melhoria na nutrição das plantas, atuando assim de forma positiva e eficiente para o melhor desenvolvimento da parte aérea e produtividade de grãos de soja.

Os teores de N, P, Ca, B, Mn e Zn foram influenciados pela aplicação dos resíduos. Em longo prazo, nota-se que o teor de $\mathrm{Zn}$ causa decréscimo na produção da cultura o que pode ser um indicativo de excesso. Já os teores de $\mathrm{Mg}, \mathrm{S}, \mathrm{Cu}$ e Fe não sofreram influência das doses. Os teores de $\mathrm{K}$ diminuíram na planta com o aumento das doses dos resíduos. A produção de grãos de soja aumentou com o aumento das doses dos resíduos, exceto para dose $8 \mathrm{Mg} \mathrm{ha}^{-1}$ do resíduo LB.

\section{Agradecimentos}

À FAPESP - Fundação de Amparo à Pesquisa do Estado de São Paulo (nº de registro: 2011 / 12189-5) e Coordenação de Aperfeiçoamento de Pessoal de Nível Superior (CAPES) pelo apoio financeiro desta pesquisa.

\section{Referências Bibliográficas}

Andrade, C.A., Boeira, R.C., Pires, A.M.M. 2010. Nitrogênio presente em lodo de esgoto e a Resolução n. ${ }^{\circ} 375$ do Conama. In: Coscione, A.R.; Nogueira, T.A.R.; Pires, A.M.M. Uso agrícola de lodo de esgoto: Avaliação após a Resolução $n^{\circ}$ 375 do Conama. Editora FEPAF, Botucatu, pp.157-170.

Corrêa, J.C.; Büll, L.T.; Crusciol, C.A.C; Marcelino, R.; Mauad, M. Correção da acidez e mobilidade de íons em Latossolo com aplicação superficial de escória, lama cal, lodos de esgoto. Pesquisa Agropecuária Brasileira, v.42, p.13071317,2007

Corrêa, J.C.; Büll, L.T.; Paganini, W.S.; Guerrini, I.A. 2008a. Disponibilidade de metais pesados em Latossolo com aplicação superficial de escória, lama cal, lodos de esgoto. Pesquisa Agropecuária Brasileira, 43, pp.411-413.

Corrêa, J.C.; Büll, L.T.; Crusciol, C.A.C.; Tecchio, M.A. 2008b. Aplicação superficial de escória, lama cal, lodos de esgoto e calcário na cultura da soja. Pesquisa Agropecuária Brasileira, Brasília, 43, 9, pp.1209-1219.

Corrêa, J.C.; Büll, L.T.; Crusciol, C.A.C.; Moraes, M.H. 2009. Alteração de atributos físicos em latossolo com aplicação superficial de escória de aciaria, lama cal, lodos de esgoto e calcário. Revista Brasileira de Ciência do Solo, Viçosa, 33, 2, pp. 263-272.

Eriksson, A.K.; Gustafsson, J.P.; Hesterberg, D. 2015. Phosphorus speciation of clay fractions from long-term fertility experiments in Sweden. Geoderma, 241-242:68-74.

Fernandez, F.A.; Büll, L.T.;Corrêa, J.C.; Crespam, D.R. 2009. Influência de silicato e calcário na decomposição de resíduos culturais e disponibilidade de nutrientes ao feijoeiro. Revista Brasileira de Ciência do Solo, Viçosa, 33, 4, pp. 935-945.

Miggiolaro, A.E.; Antonangelo, J.A.; Fernandes, D.M.; Büll, L.T.; Freitag, E.E. 2014. Diagnose foliar da cultura da soja em função da aplicação superficial de lodos de esgoto e resíduos industriais sob diferentes doses em sistema plantio direto. Journal of Agronomic Sciences, Umuarama, 6, 1, pp. 258-273.

Malavolta, E.; Vitti, G.C.; Oliveira, S.A. 1997. Avaliação do estado nutricional das plantas: princípios e aplicações, 2. ed. Potafos, Piracicaba, 319 pp.

Malavolta, E. 2006. Manual de Nutrição Mineral de Plantas. Agronômica Ceres, São Paulo, 638 pp.

Mascarenhas, H.A.A.; Esteves, J.A.F.; Wutke, E.B.; Reco, P.C.; Leão, P.C.L. 2013. Deficiência e toxicidade visuais de nutrientes em soja. 10, 2 .

Mascarenhas, H.A.A.; Tanaka, R.T.; Carmello, Q.A.C.; Gallo, P.B.; Ambrosano, G.M.B. 2000. Calcário e potássio para a cultura da soja. Scientia Agricola, 57, 3, pp. 445-449.

Nascimento, C.W.A.; Fontes, R.L.F. 2004a. Correlação entre características de Latossolos e parâmetros de equações de adsorção de cobre e zinco. Revista Brasileira de Ciência do Solo, Viçosa, 28, 6, pp. 965-971. 
Nascimento, C.W.A.; Barros, D.A.S.; Melo, E.E.C.; Oliveira, A.B. 2004b. Alterações Químicas em solos e crescimento de milho e feijoeiro após aplicação de lodo de esgoto. Revista Brasileira de Ciência do Solo, Viçosa, 21, 2, pp. 385-392.

Prado, R.M.; Fernandes, F.M. 2001. Eficiência da escória de siderurgia em latossolo vermelho na nutrição e na produção de matéria seca de cana-de-açúcar cultivada em vaso. STAB Açúcar, Álcool Subprodutos, Piracicaba, 19, pp. 26-29.

Prado, R.M.; Coutinho, E.E.M.; Roque, C.G.; Villar, M.L.P. 2002. Avaliação da escória de siderurgia e de calcários como corretivos da acidez do solo no cultivo da alface. Pesquisa Agropecuária Brasileira, Brasília, 37, 4, pp. 539-546.

Prado, R.M.; Fernandes, F.M.; Natale, W. 2003. Efeito residual da escória de siderurgia como corretivo de acidez do solo na soqueira de cana-de-açúcar. Revista Brasileira de Ciência do Solo, Viçosa, 27, 2, pp. 287-296.

Prado, R.M.; Natale, W.; Fernandes, F.M.; Corrêa, M.C.M. 2004. Reatividade de uma escória de siderurgia em um Latossolo Vermelho Distrófico. Revista Brasileira de Ciência do Solo, Viçosa, 28, 1, pp.197-205.
Raij, B. Van.; Cantarella, H.; Quaggio, J.A.; Furlani, A.M.C. 1997. Recomendações de adubação e calagem para o estado de São Paulo. 2. ed., Boletim Técnico do Instituto Agronômico de Campinas, Campinas, n. ${ }^{\circ}$ 100, 285 pp.

Raij, B. Van, Andrade, J.C., Cantarella, H., Quaggio, J.A. 2001. Análise química para avaliação da fertilidade de solos tropicais. Instituto Agronômico, Campinas, 285 pp.

Sample, E.C.; Soper, R.J.; Racz, G.J. 1980. Reactions of phosphate fertilizers in soils. In: Khasawneh, F.E.; Sample, E.C.; Kamprath, E.J., editors, The role of phosphorus in agriculture. Am. Soc. Agron., Madison, WI. 1980. pp. 263310 .

Santos, H.G. 2013. Sistema Brasileiro de Classificação de Solos. 3. ed., Embrapa , Brasília, 353 pp.

Silva, M.A.; Büll, L.T.; Miggiolaro, A.E.; Antonangelo, J.A.; Muniz, A.S. 2014. Fitodisponibilidade de metais utilizando ácidos orgânicos após sucessiva aplicação de resíduos no solo. Revista Brasileira de Engenharia Agrícola e Ambiental, Campina Grande, 18, 12, pp. 1287-1295. 